\title{
Exponentiable functors between quantaloid-enriched categories
}

\author{
Isar Stubbe*
}

February 25, 2019

\begin{abstract}
Exponentiable functors between quantaloid-enriched categories are characterized in elementary terms. The proof goes as follows: the elementary conditions translate into existence statements for certain adjoints that obey some lax commutativity; this, in turn, is precisely what is needed to prove the existence of partial products; so that the result follows from an observation by Dyckhoff and Tholen [1987].
\end{abstract}

Keywords: quantaloid, enriched category, exponentiability, partial product. MSC 2000 Classification: 06F07, 18A22, 18D05, 18D20

\section{Introduction}

The study of exponentiable morphisms in a category $\mathcal{C}$, in particular of exponentiable functors between (small) categories (i.e. Conduché fibrations), has a long history; see [Niefield, 2001] for a short account. Recently M. M. Clementino and D. Hofmann [2006] found simple necessary-and-sufficient conditions for the exponentiability of a functor between $\mathcal{V}$-enriched categories, where $\mathcal{V}$ is a symmetric quantale which has its top element as unit for its multiplication and whose underlying sup-lattice is a locale. The modest aim of this short note is to prove the following characterization of the exponentiable functors between $\mathcal{Q}$-enriched categories, where now $\mathcal{Q}$ is any (small) quantaloid, thus considerably generalizing the aforementioned result of [Clementino and Hofmann, 2006].

Theorem 1.1 A functor $F: \mathbb{A} \longrightarrow \mathbb{B}$ between $\mathcal{Q}$-enriched categories is exponentiable if and only if the following two conditions hold:

1. for every $a, a^{\prime} \in \mathbb{A}$ and $\bigvee_{i} f_{i} \leq \mathbb{B}\left(F a^{\prime}, F a\right)$,

$$
\left(\bigvee_{i} f_{i}\right) \wedge \mathbb{A}\left(a^{\prime}, a\right)=\bigvee_{i}\left(f_{i} \wedge \mathbb{A}\left(a^{\prime}, a\right)\right)
$$

${ }^{*}$ Centro de Matemática, Universidade de Coimbra, Portugal. Email: isar@mat.uc.pt 
2. for every $a, a^{\prime \prime} \in \mathbb{A}, b^{\prime} \in \mathbb{B}, f \leq \mathbb{B}\left(b^{\prime}, F a\right)$ and $g \leq \mathbb{B}\left(F a^{\prime \prime}, b^{\prime}\right)$,

$$
(g \circ f) \wedge \mathbb{A}\left(a^{\prime \prime}, a\right)=\bigvee_{a^{\prime} \in F^{-1} b^{\prime}}\left(\left(g \wedge \mathbb{A}\left(a^{\prime \prime}, a^{\prime}\right)\right) \circ\left(f \wedge \mathbb{A}\left(a^{\prime}, a\right)\right)\right) .
$$

These conditions are "elementary" in the sense that they are simply equalities (of infima, suprema and compositions) of morphisms in the base quantaloid $\mathcal{Q}$. The second condition is precisely what [Clementino and Hofmann, 2006] had too, but they did not discover the first condition an sich: because it is obviously always true if the base category is a locale.

The proof of 1.1 goes as follows. First we translate the conditions in 1.1 into existence statements for certain adjoints obeying some lax commutativity, see section

3. Next, in section 4, we show that these latter adjoints are precisely what is needed to prove the existence of partial products in $\operatorname{Cat}(\mathcal{Q})$ over $F: \mathbb{A} \longrightarrow \mathbb{B}$. The result then follows from R. Dyckhoff and W. Tholen's [1987] observation that a morphism $f: A \longrightarrow B$ in a category $\mathcal{C}$ with finite limits is exponentiable if and only if $\mathcal{C}$ admits partial products over $f$.

Acknowledgement. I thank Maria Manuel Clementino and Dirk Hofmann for explaining me their paper [2006] and suggesting the topic of this note; and I thank them again for patiently listening to me explaining the result presented here. This work was done in the spring of 2006, during my post-doc stay at the Centre for Mathematics of the University of Coimbra.

\section{Fixing notation}

For the basics on $\mathcal{Q}$-enriched categories we refer to [Stubbe, 2005]. Here we shall just observe that $\operatorname{Cat}(\mathcal{Q})$ has pullbacks and a terminal - and therefore all finite limits [Borceux, 1994, Proposition 2.8.2] - and fix some notations.

The terminal object in $\operatorname{Cat}(\mathcal{Q})$, write it as $\mathbb{T}$, has:

- objects: $\mathbb{T}_{0}=\mathcal{Q}_{0}$, with types $t X=X$,

- hom-arrows: $\mathbb{T}(Y, X)=\top_{X, Y}=$ the top element of $\mathcal{Q}(X, Y)$.

For two functors $F: \mathbb{A} \longrightarrow \mathbb{C}$ and $G: \mathbb{B} \longrightarrow \mathbb{C}$ with common codomain, their pullback $\mathbb{A} \times_{\mathbb{C}} \mathbb{B}$ has:

- objects: $\left.\left(\mathbb{A} \times_{\mathbb{C}} \mathbb{B}\right)_{0}=\left\{(a, b) \in \mathbb{A}_{0} \times \mathbb{B}_{0}\right) \mid F a=G b\right\}$ with $t(a, b)=t a=t b$,

- hom-arrows: $\left(\mathbb{A} \times_{\mathbb{C}} \mathbb{B}\right)\left(\left(a^{\prime}, b^{\prime}\right),(a, b)\right)=\mathbb{A}\left(a^{\prime}, a\right) \wedge \mathbb{B}\left(b^{\prime}, b\right)$,

and comes with the obvious projections. All verifications are entirely straightforward. 
For an $X \in \mathcal{Q}$, the one-object $\mathcal{Q}$-category with hom-arrow $1_{X}$ is written as $*_{X}$. There is an obvious bijection between the objects of type $X$ in some $\mathcal{Q}$-category $\mathbb{B}$ and the functors from $*_{X}$ to $\mathbb{B}$. Thus, let $[b]: *_{t b} \longrightarrow \mathbb{B}$ stand for the functor "pointing at" $b \in \mathbb{B}$. Given a functor $F: \mathbb{A} \longrightarrow \mathbb{B}$ and an object $b \in \mathbb{B}$ in its codomain, we shall write $\mathbb{A}_{b}$ for the pullback

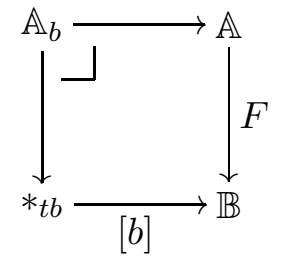

That is to say, $\mathbb{A}_{b}$ has

- objects: $\mathbb{A}_{b}=F^{-1} b=\{a \in \mathbb{A} \mid b=F a\}$, all of type $t b$,

- hom-arrows: $\mathbb{A}_{b}\left(a^{\prime}, a\right)=1_{t b} \wedge \mathbb{A}\left(a^{\prime}, a\right)$.

\section{Adjoints obeying a lax commutativity}

In this section we shall translate conditions 1.11 and 1.1 2 to existence statements of certain adjoints obeying some lax commutative diagrams.

Lemma 3.1 For a functor $F: \mathbb{A} \longrightarrow \mathbb{B}$ between $\mathcal{Q}$-categories, the following are equivalent conditions:

1. 1.1] holds,

2. for every $a, a^{\prime} \in \mathbb{A}$, the order-preserving map

$$
\downarrow \mathbb{B}\left(F a^{\prime}, F a\right) \longrightarrow \mathcal{Q}\left(t a, t a^{\prime}\right): f \mapsto f \wedge \mathbb{A}\left(a^{\prime}, a\right)
$$

has a right adjoint,

3. for every $b, b^{\prime} \in F(\mathbb{A})$, the order-preserving map

$$
\downarrow \mathbb{B}\left(b^{\prime}, b\right) \longrightarrow \operatorname{Matr}(\mathcal{Q})\left(\mathbb{A}_{b}, \mathbb{A}_{b^{\prime}}\right): f \mapsto\left(f \wedge \mathbb{A}\left(a^{\prime}, a\right)\right)_{\left(a, a^{\prime}\right) \in \mathbb{A}_{b} \times \mathbb{A}_{b^{\prime}}}
$$

has a right adjoint.

4. for every $b, b^{\prime} \in \mathbb{B}$, the order-preserving map in (2) has a right adjoint.

Proof: The equivalence of the first two statements is trivial: an order-preserving map between complete lattices has a right adjoint if and only if it preserves arbitrary 


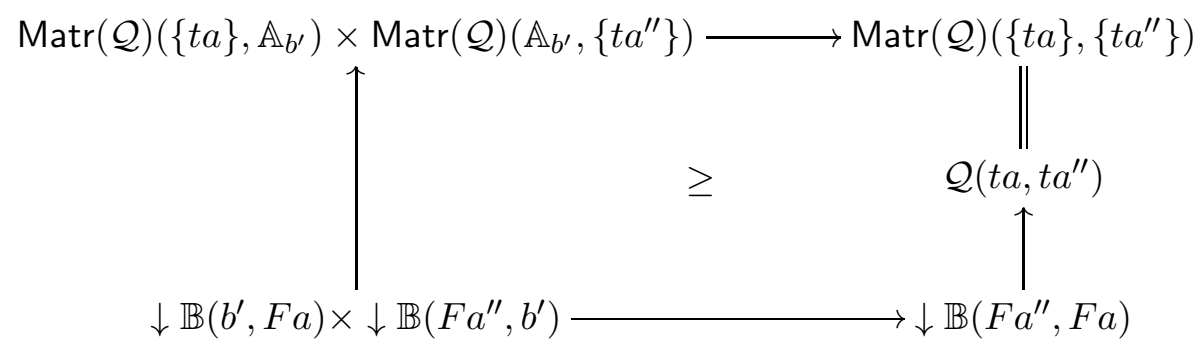

Figure 1: the diagram for $3.2-2$

suprema. Further, if we use $g \mapsto g^{F}$ as generic notation for the right adjoints to the maps in (11), then

$$
M \mapsto M^{F}:=\bigwedge\left\{M\left(a^{\prime}, a\right)^{F} \mid\left(a, a^{\prime}\right) \in \mathbb{A}_{b} \times \mathbb{A}_{b^{\prime}}\right\}
$$

is the right adjoint to the map in (2). Conversely, if $M \mapsto M^{F}$ is the right adjoint to the map in (2), then for any $a, a^{\prime} \in \mathbb{A}$

$$
g \mapsto g^{F}:=\left(T^{\left(a, a^{\prime}\right)}(g)\right)^{F}
$$

is the right adjoint to the map in (1), with $T^{\left(a, a^{\prime}\right)}(g)$ standing for the $\mathcal{Q}$-matrix from $\mathbb{A}_{b}$ to $\mathbb{A}_{b^{\prime}}$ all of whose elements are set to the top element in $\mathcal{Q}\left(t b, t b^{\prime}\right)=\mathcal{Q}\left(t a, t a^{\prime}\right)$ except for the element indexed by $\left(a, a^{\prime}\right)$ which is set to $g$. Finally, the only difference between the third and the fourth statement is that in the latter it may be that $\mathbb{A}_{b}$ or $\mathbb{A}_{b^{\prime}}$ is empty; but then $\operatorname{Matr}(\mathcal{Q})\left(\mathbb{A}_{b}, \mathbb{A}_{b^{\prime}}\right)$ is a singleton (containing the empty matrix) in which case the right adjoint to (2) always exists.

Lemma 3.2 For a functor $F: \mathbb{A} \longrightarrow \mathbb{B}$ between $\mathcal{Q}$-categories for which the equivalent conditions in 3.1 hold, the following are equivalent conditions:

1. 1.1 2 holds,

2. for every $a, a^{\prime \prime} \in \mathbb{A}$ and $b^{\prime} \in \mathbb{B}$, the diagram in figure 1, in which the horizontal arrows are given by composition (in $\operatorname{Matr}(\mathcal{Q})$, resp. $\mathcal{Q})$, the left vertical arrow is

$$
(f, g) \mapsto\left(\left(f \wedge \mathbb{A}\left(a^{\prime}, a\right)\right)_{a^{\prime} \in \mathbb{A}_{b^{\prime}}},\left(g \wedge \mathbb{A}\left(a^{\prime \prime}, a^{\prime}\right)\right)_{a^{\prime} \in \mathbb{A}_{b^{\prime}}}\right)
$$

and the right vertical arrow is a map as in (11), is lax commutative as indicated,

3. for every $b, b^{\prime \prime} \in F(\mathbb{A})$ and $b^{\prime} \in \mathbb{B}$, the diagram in figure $\mathbb{2}$, in which the horizontal arrows are given by composition and the vertical arrows are (products of) maps as in (2), is lax commutative as indicated, 


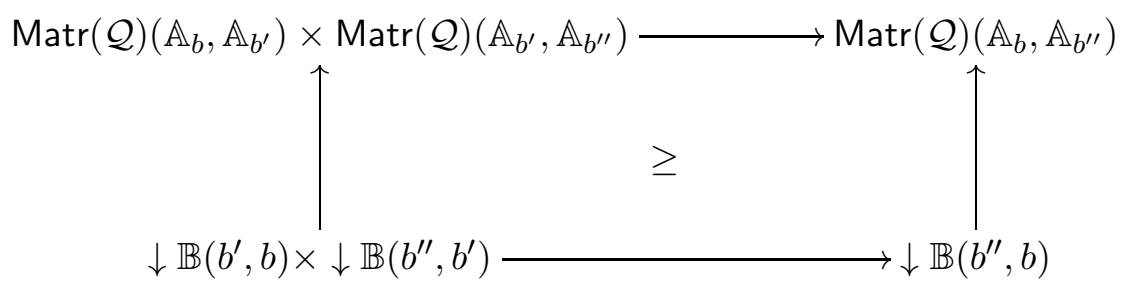

Figure 2: the diagram for $3.2-3$

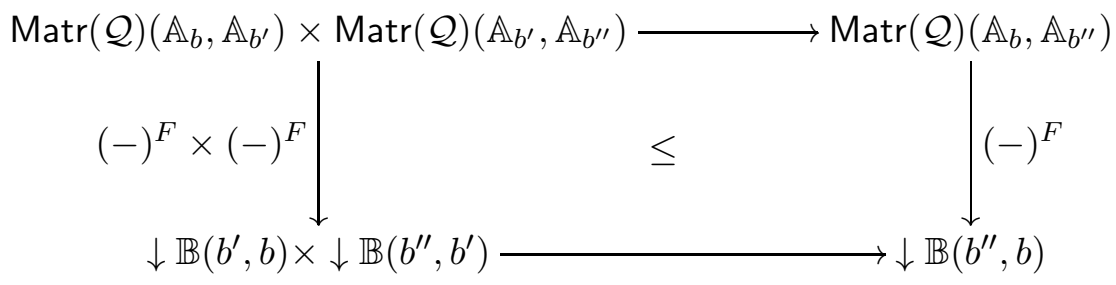

Figure 3: the diagram for 3.25

4. for every $b, b^{\prime}, b^{\prime \prime} \in \mathbb{B}$, the diagram in figure 2 , in which the horizontal arrows are given by composition and the vertical arrows are (products of) maps as in (2), is lax commutative as indicated,

5. for every $b, b^{\prime}, b^{\prime \prime} \in \mathbb{B}$, the diagram in figure 3 , in which the horizontal arrows are given by composition and the vertical arrows are instances of the adjoints to the maps as in (2), is lax commutative as indicated.

Proof: The equivalence of the first two statements is immediate; the "oplax commutativity" of the diagram in figure 1 is always true, thus explaining why in 1.1 2 there is an equality instead of an inequality. That the second and the third statement are equivalent, is because operations on a $\mathcal{Q}$-matrix are done "elementwise"; and the third and fourth are equivalent because in case $\mathbb{A}_{b}$ or $\mathbb{A}_{b^{\prime \prime}}$ is empty, $\operatorname{Matr}(\mathcal{Q})\left(\mathbb{A}_{b}, \mathbb{A}_{b^{\prime \prime}}\right)$ is a singleton, hence all is trivial. Finally, the equivalence of the two last statements follows from the respective vertical arrows being adjoint.

\section{Partial products}

In this section we complete the proof of 1.1 by establishing a link with the theory of partial products.

First recall Dyckhoff and Tholen's [1987] definition (which they gave for any morphism $f: A \longrightarrow B$ and any object $C$ in any category $\mathcal{C}$ with finite limits, but here it is for $\mathcal{Q}$-categories): the partial product of a functor $F: \mathbb{A} \longrightarrow \mathbb{B}$ with a $\mathcal{Q}$-category $\mathbb{C}$ is a $\mathcal{Q}$-category $\mathbb{P}$ together with functors $P: \mathbb{P} \longrightarrow \mathbb{B}, E: \mathbb{P} \times \mathbb{B} \mathbb{A} \longrightarrow \mathbb{C}$ such that, 


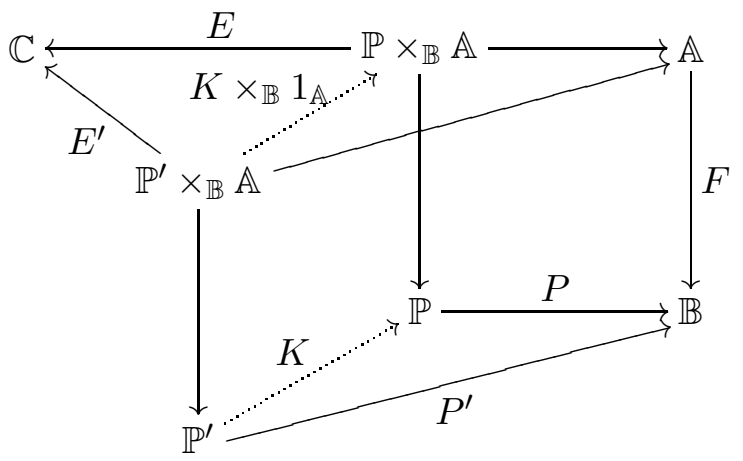

Figure 4: the definition of a partial product

for any other $\mathcal{Q}$-category $\mathbb{P}^{\prime}$ and functors $P^{\prime}: \mathbb{P}^{\prime} \longrightarrow \mathbb{B}, E^{\prime}: \mathbb{P}^{\prime} \times \mathbb{B} \mathbb{A} \longrightarrow \mathbb{C}$ there exists a unique functor $K: \mathbb{P}^{\prime} \longrightarrow \mathbb{P}$ satisfying $P \circ K=P^{\prime}$ and $E \circ\left(K \times_{\mathbb{B}} 1_{\mathbb{A}}\right)=E^{\prime}$. Figure 4 pictures the situation.

Suppose now that $F: \mathbb{A} \longrightarrow \mathbb{B}$ and $\mathbb{C}$ are given, and that we want to describe - if it exists - the partial product $\mathbb{P}$ together with its functors $P$ and $E$. Putting $\mathbb{P}^{\prime}=*_{X}$ in the diagram in figure 4 and letting $X$ range over all objects of $\mathcal{Q}$, determines at once the object-set $\mathbb{P}_{0}$ and the object-maps $P: \mathbb{P}_{0} \longrightarrow \mathbb{C}_{0}$ and $E:(\mathbb{P} \times \mathbb{B} \mathbb{A})_{0} \longrightarrow \mathbb{C}_{0}$ :

- $\mathbb{P}_{0}=\left\{(b, H) \mid b \in \mathbb{B}\right.$ and $H: \mathbb{A}_{b} \longrightarrow \mathbb{C}$ is a functor $\left.{ }^{1}\right\}$, with types $t(b, H)=t b$,

- for $(b, H) \in \mathbb{P}_{0}, P(b, H)=b$,

- for $((b, H), a) \in(\mathbb{P} \times \mathbb{B} \mathbb{A})_{0}, E((b, H), a)=H a$.

Thus we are left to find a $\mathcal{Q}$-enrichment of the object-set $\mathbb{P}_{0}$, making it a $\mathcal{Q}$-category $\mathbb{P}$ and making $P$ and $E$ functors, having the required universal property.

Lemma 4.1 Cat $(\mathcal{Q})$ admits partial products over $F: \mathbb{A} \longrightarrow \mathbb{B}$ if and only if $F: \mathbb{A} \longrightarrow \mathbb{B}$ satisfies 3.1 , and 3.25 .

Proof: Sufficiency of 3.1] 4 and 3.2] [5. Assuming 3.1] it makes sense to define

$$
\begin{aligned}
\mathbb{P}\left(\left(b^{\prime}, H^{\prime}\right),(b, H)\right):= & \mathbb{C}\left(H^{\prime}-, H-\right)^{F} \\
= & \text { the outcome of applying the right adjoint to the map } \\
& \text { in (2) on the } \mathcal{Q} \text {-matrix }\left(\mathbb{C}\left(H^{\prime} a^{\prime}, H a\right)\right)_{\left(a, a^{\prime}\right) \in \mathbb{A}_{b} \times \mathbb{A}_{b^{\prime}}}
\end{aligned}
$$

Whereas the identity inequality

$$
1_{t(b, H)} \leq \mathbb{P}((b, H),(b, H))
$$


reduces to the fact that $H: \mathbb{A}_{b} \longrightarrow \mathbb{C}$ is a functor, it is the assumed 3.2 [5 together with the composition inequality in the $\mathcal{Q}$-category $\mathbb{C}$ that assures the composition inequality

$$
\mathbb{P}\left(\left(b^{\prime \prime}, H^{\prime \prime}\right),\left(b^{\prime}, H^{\prime}\right)\right) \circ \mathbb{P}\left(\left(b^{\prime}, H^{\prime}\right),(b, H)\right) \leq \mathbb{P}\left(\left(b^{\prime \prime}, H^{\prime \prime}\right),(b, H)\right) .
$$

Moreover, this construction clearly makes $P$ and $E$ functorial:

$$
\begin{gathered}
\mathbb{P}\left(\left(b^{\prime}, H^{\prime}\right),(b, H)\right) \leq \mathbb{B}\left(b^{\prime}, b\right), \\
\mathbb{P}\left(\left(b^{\prime}, H^{\prime}\right),(b, H)\right) \wedge \mathbb{A}\left(a^{\prime}, a\right) \leq \mathbb{C}\left(H^{\prime} a^{\prime}, H a\right) \text { for any } a \in \mathbb{A}_{b} \text { and } a^{\prime} \in \mathbb{A}_{b^{\prime}} .
\end{gathered}
$$

As for the universal property, for a given $\mathcal{Q}$-category $\mathbb{P}^{\prime}$ together with functors $P^{\prime}: \mathbb{P}^{\prime} \longrightarrow \mathbb{B}$ and $E^{\prime}: \mathbb{P}^{\prime} \times \mathbb{B} \mathbb{A} \longrightarrow \mathbb{C}$, it is straightforward to verify that

$$
K: \mathbb{P}^{\prime} \longrightarrow \mathbb{P}: x \mapsto K(x):=\left(P^{\prime} x, E^{\prime}(x,-): \mathbb{A}_{P^{\prime} x} \longrightarrow \mathbb{C}: a \mapsto E^{\prime}(x, a)\right)
$$

is the required unique factorization.

Necessity of 3.1] Let $M \in \operatorname{Matr}(\mathcal{Q})\left(\mathbb{A}_{b}, \mathbb{A}_{b^{\prime}}\right)$ for some $b, b^{\prime} \in \mathbb{B}$, then consider the $\mathcal{Q}$-category $\mathbb{C}$ as follows:

- objects: $\mathbb{C}_{0}=\mathbb{A}_{b} \uplus \mathbb{A}_{b^{\prime}}$ with "inherited types",

- hom-arrows: $\mathbb{C}\left(a^{\prime}, a\right)=M\left(a^{\prime}, a\right)$ when $a \in \mathbb{A}_{b}$ and $a^{\prime} \in \mathbb{A}_{b^{\prime}}$, all endo-homarrows are identities, and all remaining hom-arrows are zero.

Assuming that the partial product $\mathbb{P}$ (together with its functors $P$ and $E$ ) exists, we get from the discussion prior to the statement of 3.2 that $\mathbb{P}_{0}$ contains in particular

$$
\left(b, H: \mathbb{A}_{b} \longrightarrow \mathbb{C}: a \mapsto a\right) \text { and }\left(b^{\prime}, H^{\prime}: \mathbb{A}_{b^{\prime}} \longrightarrow \mathbb{C}: a^{\prime} \mapsto a^{\prime}\right)
$$

Next, let $f \leq \mathbb{B}\left(b^{\prime}, b\right)$ in $\mathcal{Q}(X, Y)$ and consider the $\mathcal{Q}$-category $\mathbb{P}_{f}$ with:

- objects: $\left(\mathbb{P}_{f}\right)_{0}=\{X\} \uplus\{Y\}$ with $t X=X \in \mathcal{Q}$ and $t Y=Y \in \mathcal{Q}$,

- hom-arrows: $\mathbb{P}_{f}(Y, X)=f, \mathbb{P}_{f}(X, X)=1_{X}, \mathbb{P}_{f}(Y, Y)=1_{Y}$ and $\mathbb{P}_{f}(X, Y)=$ $0_{Y, X}$.

There is a functor $P^{\prime}: \mathbb{P}_{f} \longrightarrow \mathbb{B}: X \mapsto b, Y \mapsto b^{\prime}$, and because of the existence of the partial product, the following are now equivalent statements:

1. for all $a \in \mathbb{A}_{b}$ and $a^{\prime} \in \mathbb{A}_{b^{\prime}}, f \wedge \mathbb{A}\left(a^{\prime}, a\right) \leq M\left(a^{\prime}, a\right)$,

2. there is a functor $E^{\prime}: \mathbb{P}_{f} \times \mathbb{B} \mathbb{A} \longrightarrow \mathbb{C}:\left\{\begin{array}{l}(X, a) \mapsto a \\ \left(Y, a^{\prime}\right) \mapsto a^{\prime}\end{array}\right.$ 
3. there is a functor $K: \mathbb{P}_{f} \longrightarrow \mathbb{P}:\left\{\begin{array}{l}X \mapsto(b, H) \\ Y \mapsto\left(b^{\prime}, H^{\prime}\right)\end{array}\right.$ (with notations as in (3)),

4. $f \leq \mathbb{P}\left(\left(b^{\prime}, H^{\prime}\right),(b, H)\right)$ (again with notations as in (3) ).

Hence $\mathbb{P}\left(\left(b^{\prime}, H^{\prime}\right),(b, H)\right)$ is precisely the value on $M$ of the sought-after right adjoint to the map in (2).

Necessity of 3.2 5. Consider objects $b, b^{\prime}, b^{\prime \prime} \in \mathbb{B}$ and matrices $M \in \operatorname{Matr}(\mathcal{Q})\left(\mathbb{A}_{b}, \mathbb{A}_{b^{\prime}}\right)$ and $N \in \operatorname{Matr}(\mathcal{Q})\left(\mathbb{A}_{b^{\prime}}, \mathbb{A}_{b^{\prime \prime}}\right)$; these determine a $\mathcal{Q}$-category $\mathbb{C}$ like so:

- objects: $\mathbb{C}_{0}=\mathbb{A}_{b} \uplus \mathbb{A}_{b^{\prime}} \uplus \mathbb{A}_{b^{\prime \prime}}$ with "inherited types",

- hom-arrows: all endo-hom-arrows are identities, for $a \in \mathbb{A}_{b}, a^{\prime} \in \mathbb{A}_{b^{\prime}}, a^{\prime \prime} \in \mathbb{A}_{b^{\prime \prime}}$ put $\mathbb{C}\left(b^{\prime \prime}, b^{\prime}\right)=N\left(b^{\prime \prime}, b^{\prime}\right), \mathbb{C}\left(b^{\prime}, b\right)=M\left(b^{\prime}, b\right)$ and $\mathbb{C}\left(b^{\prime \prime}, b\right)=(N \circ M)\left(b^{\prime \prime}, b\right)$, all other hom-arrows are zero.

Assuming that the partial product of $F$ and $\mathbb{C}$ exists, the objects

$$
\left(b, H: \mathbb{A}_{b} \longrightarrow \mathbb{C}: a \mapsto a\right),\left(b^{\prime}, H: \mathbb{A}_{b^{\prime}} \longrightarrow \mathbb{C}: a^{\prime} \mapsto a^{\prime}\right) \text { and }\left(b^{\prime \prime}, H: \mathbb{A}_{b^{\prime \prime}} \longrightarrow \mathbb{C}: a^{\prime \prime} \mapsto a^{\prime \prime}\right)
$$

of the partial product $\mathbb{P}$ satisfy in particular the compostion-inequality

$$
\mathbb{P}\left(\left(b^{\prime \prime}, H^{\prime \prime}\right),\left(b^{\prime}, H^{\prime}\right)\right) \circ \mathbb{P}\left(\left(b^{\prime}, H^{\prime}\right),(b, H)\right) \leq \mathbb{P}\left(\left(b^{\prime \prime}, H^{\prime \prime}\right),(b, H)\right),
$$

saying precisely that $N^{F} \circ M^{F} \leq(N \circ M)^{F}$.

\section{Comments}

Exponentiable $\mathcal{Q}$-categories. A $\mathcal{Q}$-category $\mathbb{A}$ is exponentiable if and only if the unique functor !: $\mathbb{A} \longrightarrow \mathbb{T}$ into the terminal is exponentiable, which - as a corollary of 1.1 - easily translates into two elementary necessary-and-sufficient conditions.

Multiple pullback of partial products of $\mathcal{Q}$-arrows. With notations as in the proof of 3.1 given objects $b, b^{\prime} \in \mathbb{B}$ and a $\mathcal{Q}$-matrix $M: \mathbb{A}_{b} \longrightarrow \mathbb{A}_{b^{\prime}}$, the $\mathcal{Q}$-arrow $M\left(a^{\prime}, a\right)^{F}$ is precisely the partial product in $\mathcal{Q}\left(t a, t a^{\prime}\right)$ as suggested by the diagram in figure 5. And the $\mathcal{Q}$-arrow

$$
M^{F}=\bigwedge\left\{M\left(a^{\prime}, a\right)^{F} \mid\left(a, a^{\prime}\right) \in \mathbb{A}_{b} \times \mathbb{A}_{b^{\prime}}\right\}
$$

which results from applying the right adjoint to the map in (2) to the matrix $M$, is then the multiple pullback in $\mathcal{Q}\left(t a, t a^{\prime}\right)$ of these partial products. In other words, condition 1.11 says something about the existence of certain multiple pullbacks of partial products in the hom-sup-lattices of $\mathcal{Q}$. 


$$
\begin{aligned}
M\left(a^{\prime}, a\right) \geq M\left(a^{\prime}, a\right)^{F} \wedge \mathbb{A}\left(a^{\prime}, a\right) & \leq \mathbb{A}\left(a^{\prime}, a\right) \\
\mid \wedge & \mid \wedge \\
M\left(a^{\prime}, a\right)^{F} & \leq \mathbb{B}\left(F a^{\prime}, F a\right)
\end{aligned}
$$

Figure 5: another partial product

On the construction of $\mathcal{Q}$-categories. In the proof of 4.1 we build $\mathcal{Q}$-categories from given $\mathcal{Q}$-matrices, in such a way that certain hom-arrows of the category are precisely the elements of the matrix. A $\mathcal{Q}$-matrix is exactly a distributor between discrete $\mathcal{Q}$-categories, and in fact this construction of a $\mathcal{Q}$-category can be carried out for any given distributor. To see this, let $\Phi: \mathbb{A} \multimap \mathbb{B}$ be a distributor; consider now the endo- $\mathcal{Q}$-matrix

$$
\left(\begin{array}{cc}
\mathbb{A} & \Phi \\
0_{\mathbb{A}, \mathbb{B}} & \mathbb{B}
\end{array}\right)
$$

on the $\mathcal{Q}$-typed set $\mathbb{A}_{0} \uplus \mathbb{B}_{0}$ : on easily sees that this is a monad, i.e. a $\mathcal{Q}$-category. That is to say, this $\mathcal{Q}$-category, call it $[\Phi]$, has:

- objects: $[\Phi]_{0}=\mathbb{A}_{0} \uplus \mathbb{B}_{0}$ with "inherited types",

- hom-arrows: for $a, a^{\prime} \in \mathbb{A}_{0}$ and $b, b^{\prime} \in \mathbb{B}_{0}$, put $[\Phi]\left(a^{\prime}, a\right)=\mathbb{A}\left(a^{\prime}, a\right),[\Phi]\left(b^{\prime}, b\right)=$ $\mathbb{B}\left(b^{\prime}, b\right),[\Phi](b, a)=\Phi(b, a)$ and $[\Phi](a, b)=0_{t a, t b}$.

This construction is of independent interest: writing $S_{\mathbb{A}}: \mathbb{A} \longrightarrow[\Phi]$ and $S_{\mathbb{B}}: \mathbb{B} \longrightarrow[\Phi]$ for the obvious inclusions, $\Phi$ is precisely the distributor "represented by" these functors in a universal way: $\Phi=[\Phi]\left(S_{\mathbb{B}}-, S_{\mathbb{A}}-\right.$ ). (See also footnote 3 in [Stubbe, 2005].)

\section{References}

[1] [Francis Borceux, 1994] Handbook of Categorical Algebra I, Cambridge University Press, Cambridge.

[2] [Maria Manuel Clementino and Dirk Hofmann, 2006] Exponentiation in $\mathcal{V}$ categories, Topology Appl., to appear.

[3] [Roy Dyckhoff and Walter and Tholen, 1987] Exponentiable morphisms, partial products and pullback complements, J. Pure Appl. Algebra 49, pp. 103-116.

[4] [Susan Niefield, 2001] Exponentiable morphisms: posets, spaces, locales, and Grothendieck toposes, Theory Appl. Categories 8, pp. 16-32. 
[5] [Isar Stubbe, 2005] Categorical structures enriched in a quantaloid: categories, distributors, functors, Theory Appl. Categories 14, pp. 1-45 